\title{
Scaling near random criticality in two-dimensional Dirac fermions
}

\author{
Y. Morita and Y. Hatsugai \\ Department of Applied Physics, University of Tokyo, 7-3-1 Hongo Bunkyo-ku, Tokyo 113, Japan
}

(Received 29 May 1998)

\begin{abstract}
Recently the existence of a random critical line in two-dimensional Dirac fermions was confirmed. In this paper, we focus on its scaling properties, especially in the critical region. We treat Dirac fermions in two dimensions with two types of randomness, a random site (RS) model and a random hopping (RH) model. The RS model belongs to the usual orthogonal class and all states are localized. For the RH model, there is an additional symmetry expressed by $\{\mathcal{H}, \gamma\}=0$. Therefore, although all nonzero energy states localize, the localization length diverges at the zero energy. In the weak localization region, the generalized Ohm's law in fractional dimensions, $d^{*}(<2)$, has been observed for the RH model. [S0163-1829(98)03635-2]
\end{abstract}

The study of quantum phase transitions driven by randomness has a long history. In 1958, Anderson discussed the absence of diffusion in random systems. ${ }^{1}$ In 1979 , scaling arguments by Thouless et al. ${ }^{2}$ were further developed. ${ }^{3}$ The dimensionless conductance $g(L)$ is treated as the only scaling parameter, where $L$ is the linear system size. When the randomness is weak $(g \gg 1)$, the metallic behavior of the $d$-dimensional system implies $g(L)=\sigma L^{d-2}$ with conductivity $\sigma$ ("the Ohm's law"). On the other hand, when the randomness is strong $(g \ll 1)$, the wave function is exponentially localized, which brings $g=g_{0} \exp (-L / \xi)$. In the scaling argument, the beta function $\beta=d \ln g / d \ln L=\beta(g)$ plays a central role. In the above two asymptotic limits, the explicit forms are given by $\beta(g)=(d-2)+c_{1} / g+O\left(1 / g^{2}\right)$ $(g \gg 1)$ and $\beta(g)=\ln \left(g / g_{0}\right)(g \ll 1)$. It means that all states are localized and the quantum phase transition is absent in one and two dimensions. However, random critical points can appear in two dimensions and much effort has been devoted to the study. One of the cases is quantum Hall systems where the time-reversal symmetry is broken and they belong to the unitary class. ${ }^{4}$ In spite of the experimental and theoretical implications, since it is beyond the weak-coupling regime, it is still difficult to understand the critical phenomena. In this paper, another example of the random criticality in two dimensions, which has been discovered recently, is studied in detail. The properties on the critical point $(E=0)$ have been studied intensively. ${ }^{5}$ Here the scaling properties and the weak localization effect are studied in a critical region $(|E| \gtrsim 0)$. This is a quantum critical phenomena near the random critical line. It brings a novel weak localization effect ("Ohm's law in fractional dimensions").

Recently, the possible existence of a random critical line in two dimensions was suggested ${ }^{6}$ and Dirac fermions with link-type randomness in two dimensions were studied numerically. ${ }^{7,8}$ The random Dirac fermions were realized on a square lattice by the $\pi$-flux model $^{9}$ with random hopping. Our model preserves the time-reversal symmetry and belongs to the orthogonal class. The zero-energy states do not localize but become critical, which can be a prototype of critical states in two dimensions. The density of states is $\sim|E|$ without randomness, and becomes $\sim|E|^{\alpha}$ in the presence of randomness. The singularity is closely related to the appearance of critical states. Similar phenomena were found in different models, ${ }^{10,11}$ where the density of states has a singularity and nonlocalized states appear in two dimensions.
The exponent $\alpha$ changes with strength of the randomness. It implies the existence of the random critical line, which is comparable with other critical lines as the TomonagaLuttinger liquid in one-dimensional quantum systems. The stability of the zero-energy states against the random hopping is due to an additional symmetry of the Hamiltonian. The random hopping preserves the symmetry in contrast to the site-type randomness. This symmetry appears as a sign change of the Hamiltonian under the transformation $c_{j} \rightarrow$ $(-1)^{j_{x}+j_{y}} c_{j}$. The corresponding symmetry in an effective field theory is denoted by $\{\mathcal{H}, \gamma\}=0$ with a $4 \times 4$ matrix $\gamma^{7,8}$ One of the possible scenarios proposed in Ref. 8 is that all nonzero energy states are localized and the zero-energy states are just on the critical point. Unfortunately, since the localization length near the zero energy is very large and beyond numerically available system sizes, no direct argument has ever been given for the scenario. In this paper, we treat Dirac fermions with two types of randomness in the light of the scaling. Support for the above scenario is given and weak localization effect near the random criticality is discussed.

We study Dirac fermions with two types of randomness: (i) random site (RS) model and (ii) random hopping (RH) model. The Hamiltonian is given by

$$
H=\sum_{i=j \pm(1,0)}(-1)^{j_{y}} c_{i}^{\dagger} t_{i j} c_{j}+\sum_{i=j \pm(0,1)} c_{i}^{\dagger} t_{i j} c_{j}+\sum_{i} c_{i}^{\dagger} V_{i} c_{i}
$$

with (i) RS model: $t_{i j}=1, V_{i}=R\left(W_{1}\right)$ and (ii) RH model: $t_{i j}=t_{j i}=1+R\left(W_{2}\right), V_{i}=0$, where $R(W)$ 's are uniform random numbers between $[-W, W]$. Although we present data with $W_{1}=2.3$ and $W_{2}=1.0$, the qualitative feature does not depend on the strength of randomness apart from finite-size effects.

In the absence of randomness, i.e., $t_{i j}=1$ and $V_{i}=0$, the model is a tight-binding model with half a flux (" $\pi$ flux") per plaquette. 9 There are two energy bands on the magnetic Brillouin zone $[-\pi, \pi) \times[0, \pi)$, which touch at two momenta. Near the two momenta $\mathbf{k}^{i}(i=1,2)$, where the energy gap closes, the low-lying excitations are described by massless Dirac fermions in two dimensions. The effective Hamiltonian is given by $\mathcal{H}$ pure $=2 i \int d \mathbf{x} \Psi \dagger(\mathbf{x})[(\sigma 3 \otimes \sigma 1) \partial x+(I$ 
$\otimes \sigma 3) \partial \mathrm{y}] \Psi(\mathbf{x})$, where $\Psi(\mathbf{x})$ is a four-component spinor. When the Fermi energy lies at zero energy, that is, all the negative-energy eigenstates are filled, the Hall conductivity $\sigma_{x y}$ is ill defined. The sign of mass determines the $\sigma_{x y}$ in the continuum theory. ${ }^{12-14}$ There is also the following subtlety in the tight-binding model with half a flux (" $\pi$ flux") per plaquette and the next-nearest-neighbor hopping $t^{\prime} .{ }^{15}$ The $\sigma_{x y}$ is given by $t^{\prime} /\left|t^{\prime}\right|$ and, when $t^{\prime}=0$, the system is on the transition point between states with different quantum Hall conductivity. It implies that the zero mode carries nonzero Hall conductivity.

Here we briefly review some properties of the above two random systems. The effective field theory of the RS model was discussed by mapping to the nonlinear $\sigma$ model. ${ }^{16}$ It predicts the localization of all states and finite density of states at zero energy. Recently, the question of whether the density of states at the zero energy is finite or not for random Dirac fermions has been controversial. ${ }^{17,18}$ A similar model to the RS model with dilute and strong impurities (unitary limit) was also discussed and consistent results with the effective field theory were obtained. ${ }^{19,20}$ All those results suggest that the RS model belongs to the usual orthogonal class and standard scaling arguments of the Anderson localization ${ }^{3}$ seem to be valid for the RS model. On the other hand, in the case of the RH model, nonlocalized states were discovered at zero energy ${ }^{7}$ and the density of states vanishes at the zero energy as $\sim|E|^{\alpha}{ }^{8}$ It is not only a critical point but also forms a random critical line, since the exponent $\alpha$ changes with the strength of randomness. In Ref. 6, the appearance of negative $\alpha$ was suggested for sufficiently strong randomness. However, even for strong randomness $W / t=1.0$, the exponent is still positive and the negative $\alpha$ was not observed. ${ }^{8}$ Our model may be a part of the "longer" critical line. This is analogous to the massless phase of spinless fermions with nearest-neighbor interactions, which is a part of the critical line called the Tomonaga-Luttinger liquid. It may be possible to construct models with negative $\alpha$ based on our model. ${ }^{21}$ Divergence of the localization length near the zero energy was also suggested numerically. It is not allowed in a standard scenario of the two-dimensional Anderson localization.

Let us first discuss the density of states $\rho(E)$ $=\left\langle 1 / L^{2} \Sigma_{i} \delta\left(E-E_{i}\right)\right\rangle$. We diagonalize the Hamiltonian of the RS model for finite squares of size $L^{2}=30^{2}, 40^{2}$, and $50^{2}$ and ensemble average over 10000,5000 , and 3360 realizations is performed, respectively. The finite size effect is small for the density of states and only the result for $L^{2}$ $=50^{2}$ is shown in Fig. 1. The result for the RH model is also shown for comparison. ${ }^{8}$ Finite density of states at zero energy is created for the RS model, $\rho(E=0) \neq 0$. It is in contrast to the RH model, where the density of states vanishes as $\sim|E|^{\alpha}, \rho(E=0)=0$. The difference may be related to the presence of random criticality in the RH model, which will be discussed later.

Next let us discuss scaling properties of the Thouless number $g(E)=V(E) / \Delta(E)$, where $V(E)$ is an energy shift obtained by replacing periodic boundary condition with antiperiodic boundary condition in one direction and $\Delta(E)$ is a local mean level spacing near the energy $E$. The Thouless number $g(E)$ tells us how the wave function is extended in the space.

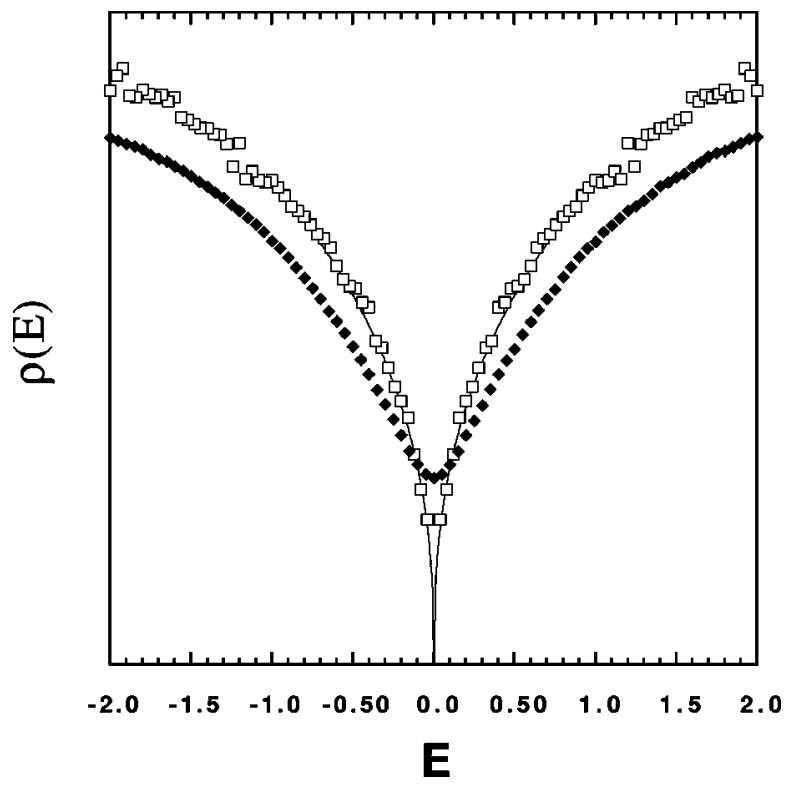

FIG. 1. Density of states for the RS model (black) and the RH model (white), where $W_{1}=2.3, W_{2}=1.0$, and $L^{2}=50^{2}$. A finite width $\delta=0.02$ is given to the delta functions, although the results do not seriously depend on the small change of $\delta$. The line is a guide for the eyes; it is $\sim|E|^{0.39}$.

Numerical results for the $g(E)$ are shown in Fig. 2. They are shown for $L^{2}=50^{2}$ and an average within an energy window is also performed together with the ensemble average. The results suggest that, in both cases, the localization length grows near the zero energy. The difference is that the growth is "singular" for the RH model, which is related to the presence of random criticality in the RH model. Although the difference is clear between the RS model and the RH model, it is crucial to apply scaling arguments to obtain definite results, which will be given below.

In the following, we assume $g=g(L, E, W)=F[y$ $=L / \xi(E, W)]$ with the localization length $\xi$, which means

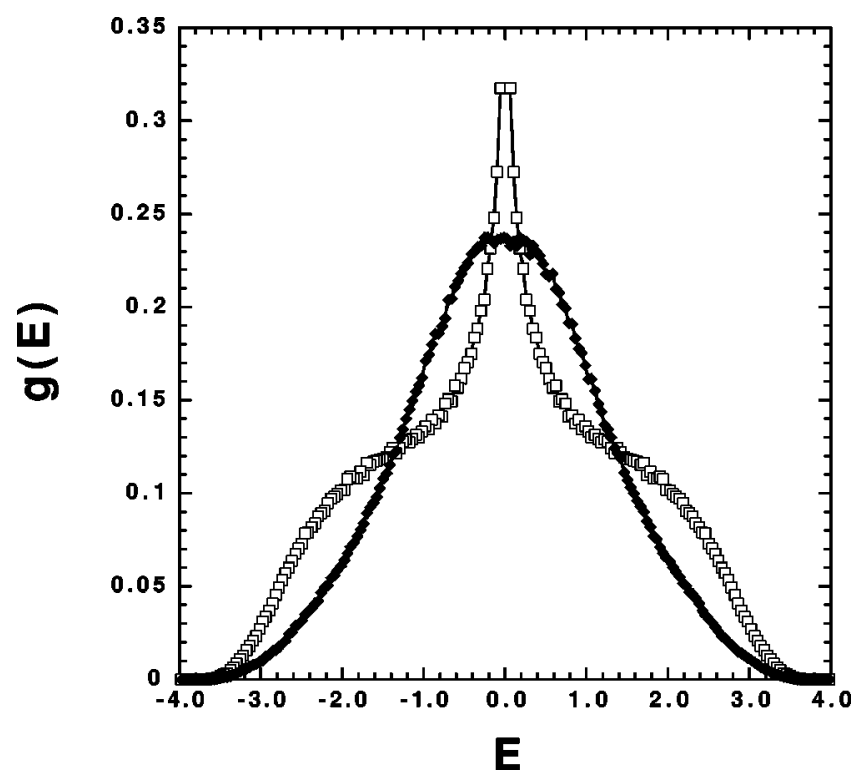

FIG. 2. The Thouless number $g(E, L)$ for the RS model (black) and the RH model (white), where $W_{1}=2.3, W_{2}=1.0$, and $L^{2}$ $=50^{2}$. 


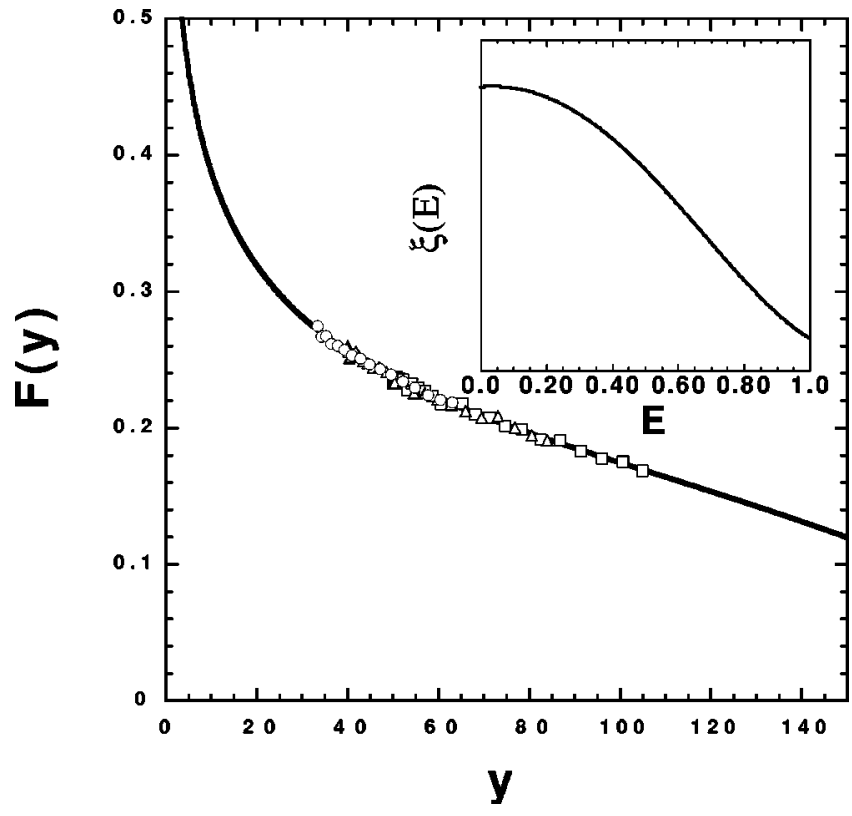

FIG. 3. Scaling function $F$ and the localization length $\xi(E)$ for the RS model. The data are $g(E, L)$ near zero energy with $W_{1}$ $=2.3, L=30,40$, and 50, and different symbols correspond to different $L$ 's. The localization length $\xi(E)$ is $\xi(E) /|E|^{\beta}=1+0.057 \epsilon$ $-0.78 \epsilon^{2}-0.32 \epsilon^{3}+0.51 \epsilon^{4}$, where $\beta=0$ and $\epsilon=|E|$. The scaling function is $F(y)=\ln (y / 230)\left(-0.12-0.00060 y+0.0000032 y^{2}\right)$. The curve beyond the data points is a guide for the eyes.

$g(L, E, W)$ 's with different $L$ and $E$ are on a single smooth curve $g=F(y)$ using the localization length $\xi(E, W)$ (scaling hypothesis). ${ }^{3,22} \mathrm{We}$ assume a functional form of the $\xi$ as

$$
\xi(E, W)=|E|^{\beta(W)} \bar{\xi}(|E|, W)
$$

with a smooth function $\bar{\xi}(\epsilon, W)=1+\xi_{1}(W) \epsilon+\cdots$. The $\xi_{n}$ 's are chosen so that $g(L, E, W)$ 's with different $L$ and $E$ are on a single smooth curve $g=F(y)$. Here the localization length $\xi(E, W)$ is introduced to define a dimensionless parameter $y[=L / \xi(E, W)]$ and is determined by the scaling hypothesis. It is related to the usual localization length $\xi_{\text {loc }}(E, W)$ of the exponentially localized wave function $|\psi(\mathbf{x}, E, W)|$ $\sim \exp \left(-\left|\mathbf{x}-\mathbf{x}_{0}\right| / \xi_{\text {loc }}(E, W)\right)$ as $\xi_{\text {loc }}(E, W)=c(W) \xi(E, W)$. Fitting our numerical results, we obtain

$$
\begin{gathered}
\beta=0 \text { for the RS model (Fig. 3), } \\
\beta=-0.75 \text { for the RH model (Fig. 4), }
\end{gathered}
$$

where $W_{1}=2.3$ for the RS model and $W_{2}=1.0$ for the $\mathrm{RH}$ model. This implies that (i) RS model: all states are localized, and (ii) RH model: all nonzero energy states are localized with the localization length $\xi(E, W)$, which is diverging as $E \rightarrow 0$, and the zero-energy states are just on a critical point.

Let us discuss the above results in the light of the scaling. Assume that the $\beta$ function for the Thouless number $g$ obeys the scaling form, i.e., $\beta=d \ln g / d \ln L=\beta(g)$. For an almost metallic state, i.e., $g \gg 1$, we expect that $\Delta(E) \sim 1 / L^{d}$ and $V(E) \sim 1 / L^{2}$ due to the level repulsion [note that $V(E)$ $\sim 1 / L$ for pure systems, since there is no level repulsion].

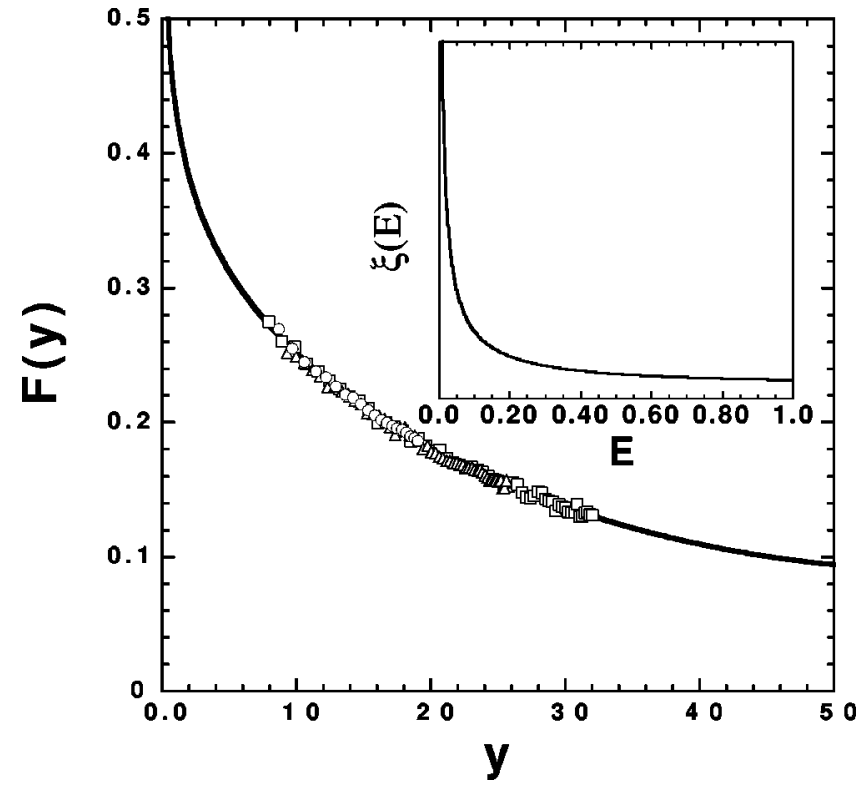

FIG. 4. Scaling function $F$ and the localization length $\xi(E)$ for the RH model. The data are $g(E, L)$ near the zero energy with $W_{2}$ $=1.0, L=30,40$, and 50, and different symbols correspond to different $L$ 's. $\xi(E) /|E|^{\beta}=1+0.47 \epsilon-0.0057 \epsilon^{2}+0.20 \epsilon^{3}-0.10 \epsilon^{4}$, where $\beta=-0.75$ and $\epsilon=|E|$. The scaling function is $F(y)$ $=y^{-0.15}\left(0.44-0.0097 y+0.000085 y^{2}\right)$. The curve beyond the data points is a guide for the eyes.

Therefore $g \sim L^{d-2}$ and $\beta(g)=(d-2)+c_{1} / g+O\left(1 / g^{2}\right)$ for large $g$. In particular, for $d=2, \beta(g)=c_{1} / g+O\left(1 / g^{2}\right)$ and $g \sim \ln (L / \xi)$ for large $g$. We confirmed that the results of the RS model can be fitted to this form, which is consistent with usual scaling arguments of the Anderson localization ${ }^{3}$ (see Fig. 3). On the other hand, although states near zero energy in the RH model are localized in the thermodynamic limit, they behave as critical states due to the large localization length beyond the available system size. Then we can expect the $g$ behaves as $\sim(L / \xi)^{\gamma}(\gamma<0)$ for the available system size. The results for the RH model are consistent with this discussion (see Fig. 4). Note that, since $g \sim \exp (-L / \xi)$ for large $L / \xi$, there is a correction in $L / \xi$ to the above expression, which is assumed to be $g=(L / \xi)^{\gamma}\left(g_{0}+g_{1} L / \xi+\cdots\right)$. We obtained good agreement with this consideration for the RH model.

Here we define an anomalous dimension $d^{*}$ as

$$
d^{*}=\lim _{g \rightarrow \infty} \beta(g)+2=\gamma+2<2
$$

for states in a critical region in two dimensions. It means $g$ $\simeq \sigma L^{d^{*-2}}$ in the weak localization regime $(g \gg 1)$. This is a generalized Ohm's law in fractional dimensions between 1 and 2. For example, we obtain $d^{*} \simeq 1.8$ in the RH model with $W_{2}=1.0$. The emergence of this anomalous dimension $d^{*}$ may be due to the multifractal nature of the zero-energy wave function in the RH model. ${ }^{7}$

Y.H. was supported in part by a Grant-in-Aid from the Ministry of Education, Science and Culture of Japan and also by the Kawakami Memorial Foundation. The computation in this work has been done using the facilities of the Supercomputer Center, ISSP, University of Tokyo. 
${ }^{1}$ P. W. Anderson, Phys. Rev. 109, 1492 (1958).

${ }^{2}$ J. T. Edwards and D. J. Thouless, J. Phys. C 5, 807 (1972); D. C. Licciardello and D. J. Thouless, ibid. 8, 4157 (1975).

${ }^{3}$ E. Abrahams, P. W. Anderson, D. C. Licciardello, and T. V. Ramakrishnan, Phys. Rev. Lett. 42, 673 (1979).

${ }^{4}$ B. Huckestein, Rev. Mod. Phys. . 67, 357 (1995).

${ }^{5}$ I. Kogan, C. Mudry, and A. M. Tsvelik, Phys. Rev. Lett. 77, 707 (1996); C. Chamon, C. Mudry, and X.-G. Wen, ibid. 77, 4194 (1996); J.-S. Caux, N. Taniguchi, and A. M. Tsvelik, ibid. 80, 1276 (1998); K. Ziegler, ibid. 80, 3113 (1998).

${ }^{6}$ A. Ludwig, M. Fisher, R. Shankar, and G. Grinstein, Phys. Rev. B 50, 7526 (1994).

${ }^{7}$ Y. Hatsugai, X. G. Wen, and M. Kohmoto, Phys. Rev. B 56, 1061 (1997).

${ }^{8}$ Y. Morita and Y. Hatsugai, Phys. Rev. Lett. 79, 3728 (1997).

${ }^{9}$ I. Affleck and J. B. Marston, Phys. Rev. B 37, 3774 (1988).

${ }^{10}$ R. Gade and F. Wegner, Nucl. Phys. B 360, 213 (1991); R. Gade, ibid. 398, 499 (1993).

${ }^{11}$ S. Hikami, M. Shirai, and F. Wegner, Nucl. Phys. B 408, 415 (1993).
${ }^{12}$ S. Deser, R. Jackiw, and S. Templeton, Ann. Phys. (N.Y.) 140, 372 (1982).

${ }^{13}$ A. Niemi and G. W. Semenoff, Phys. Rev. Lett. 51, 2077 (1983).

${ }^{14}$ A. N. Redlich, Phys. Rev. D 29, 2366 (1984).

${ }^{15}$ Y. Hatsugai and M. Kohmoto, Phys. Rev. B 42, 8282 (1990).

${ }^{16}$ M. P. A. Fisher and E. Fradkin, Nucl. Phys. B 251, 457 (1985).

${ }^{17}$ A. A. Nersesyan, A. M. Tsvelik, and F. Wenger, Phys. Rev. Lett. 72, 2628 (1994); A. A. Nersesyan and A. M. Tsvelik, ibid. 78, 3981 (1997).

${ }^{18}$ K. Ziegler, M. H. Hettler, and P. J. Hirschfeld, Phys. Rev. Lett. 77, 3013 (1996); 78, 3982 (1997).

${ }^{19}$ P. A. Lee, Phys. Rev. Lett. 71, 1887 (1993).

${ }^{20}$ Y. Hatsugai and P. A. Lee, Phys. Rev. B 48, 4204 (1993).

${ }^{21}$ Y. Morita and Y. Hatsugai (unpublished).

${ }^{22}$ Here we fixed strength of randomness and confirmed that $g(L, E, W)$ 's with different $L$ and $E$ are on a universal curve $g$ $=F(y)$. The form of $g=F(y)$ and $\beta$ depend on the strength of randomness. 